\section{J.-P. Digard ${ }^{1}$ \\ E. Landais ${ }^{2}$ \\ Ph. Lhoste ${ }^{3}$}

\title{
La crise des sociétés pastorales. Un regard pluridisciplinaire
}

DIGARD (J.-P.), LANDAIS (E.), LHOSTE (Ph.). La crise des sociétés pastorales. Un regard pluridisciplinaire. Revue Élev. Méd. vét. Pays trop., $1993,46(4): 683-692$

Cette recherche, fruit d'un échange entre un anthropologue spécialiste des sociétés pastorales et deux zootechniciens autour du thème de l'avenir des peuples pasteurs, a pour ambition de fournir au lecteur un cadre de référence pour l'analyse des manifestations locales de la crise généralisée des sociétés pastorales à travers le monde. Après avoir défini le "fait pastoral" et rappelé les fondements de la classification des différentes formes de pastoralisme, les auteurs réfutent quelques idées reçues : le pastoralisme nomade n'est pas la survivance d'une activité archaïque, mais une forme d'adaptation qui conserve sa pertinence technique ; les formations végétales des parcours exploités sont la plupart du temps anthropiques, et le maintien du pastoralisme est la condition de leur reproduction ; les sociétés pastorales, loin d'être autarciques, reposent au contraire sur une économie ouverte ; la prétendue "indolence" des pasteurs est un mythe qui repose sur une méconnaissance du travail pastoral. Ils dressent ensuite un rapide panorama des caractères communs et des spécificités des sociétés pastorales (la place centrale de l'élevage dans le champ économique, social et culturel ; le caractère guerrier ; les modes de gestion de l'espace et les pratiques pastorales) avant de décrire deux processus qui jouent un rôle majeur dans la crise actuelle : la marginalisation politique, économique, socio-culturelle et foncière des pasteurs ; la dégradation écologique des zones pastorales.

Mots clés : Pastoralisme - Société pastorale - Éleveur - Pâturages Parcours - Transhumance - Nomadisme - Système agropastoral - Economie de l'élevage - Environnement.

\section{INTRODUCTION}

Partout, les sociétés pastorales sont plongées dans une crise dont nul ne peut ignorer la gravité. Cependant, son analyse reste malaisée pour qui manque de recul pour en saisir la généralité, de repères pour en comprendre la signification historique, et de références pour interpréter celles de ses manifestations que sa formation ne l'a pas préparé à évaluer. Car cette crise de société prend des aspects politiques, économiques, démographiques, fonciers et écologiques.

Ce travail est le fruit d'un échange entre un anthropologue spécialiste des sociétés pastorales et deux zootechniciens autour du thème de l'avenir des peuples pasteurs, à l'occasion d'un récent colloque (63). Elle a pour ambition de fournir au lecteur un cadre de référence pour

\section{CNRS, 27 rue Paul-Bert, 94204 Ivry sur Seine, France.}

2. INRA-SAD, Route de Saint-Cyr, 78026 Versailles cedex, France.

3. CIRAD-EMVT, 2477 Avenue du Val de Montferrand, BP 5035, 34032 Montpellier cedex 01, France.

Reçu le 26.11.1992, accepté le 6.9.1993. lui permettre de mieux relier et interpréter les sombres événements dont les médias se font chaque jour l'écho, de la Mauritanie à l'Afghanistan, en passant par le Mali, le Niger, le Tchad, le Soudan, la Somalie, l'Ethiopie, l'Irak, la Turquie et bien d'autres lieux... It n'est bien sûr pas question de faire le point sur cette crise pastorale, dont l'analyse nécessiterait de beaucoup plus amples développements et ne saurait se satisfaire de propos généraux, tant les situations sont diverses. On se contentera de rappeler un certain nombre de particularités des sociétés pastorales au niveau de l'organisation, du fonctionnement, du mode de mise en valeur de l'espace et du type de relations qui en découle, en tentant de montrer comment ces caractéristiques sont liées entre elles et comment elles contribuent à engendrer un certain nombre de risques, qui jouent un rôle majeur dans le déclenchement local des crises. On s'appuyera pour cela sur un ensemble de références bibliographiques volontairement étoffé et dominé par les sciences humaines, à laquelle le lecteur pourra se reporter. Enfin, compte tenu de l'expérience des auteurs, les exemples et les références seront essentiellement tirés d'Afrique et du Moyen-Orient.

\section{LE PASTORALISME : ÉLÉMENTS DE DÉFINITION}

\section{Un soubassement technique}

Une "formule technique" commune définit le fait pastoral (BONTE) (15). Elle est mise en oeuvre à travers des modes d'élevage très extensifs vis-à-vis de la terre, fondés sur l'exploitation par des troupeaux d'herbivores domestiques des ressources fourragères spontanées offertes par les parcours naturels, qui sont consommées sur pied. Ceci impose d'importants déplacements des troupeaux - et donc des hommes - à la recherche de pâturages, d'eau et souvent de sel. La mobilité qui en résulte, avec ses multiples modalités, est un trait fondamental des systèmes pastoraux.

Pour les sciences de l'Homme, le pastoralisme est donc un phénomène à trois composantes essentielles:

- un milieu physique ;

- un mode d'élevage qui détermine une forme d'occupation et d'exploitation particulières de ce milieu ;

- une organisation sociale qui en permet l'adaptation permanente aux contraintes externes et assure de ce fait la reproduction du système pastoral. 
Une fois n'est pas coutume, ce point de vue est parfaitement compatible avec celui des chercheurs techniciens, qui développent à propos des systèmes pastoraux des modèles à trois "pôles" (39) : l'homme (berger, groupe familial, société pastorale) ; l'animal (troupeau, population, peuplement) ; le territoire (milieu, ressources fourragères). L'outil central de la "formule technique" du pastoralisme est constitué, selon BONTE (15) de l'ensemble des méthodes utilisées par les pasteurs pour organiser leurs animaux en troupeaux, ce que les zootechniciens désignent sous le terme de pratiques d'agrégation ou d'allotement (36)

\section{Caractères discriminants et éléments de classification}

Le souci de classer les différentes formes de pastoralisme semble beaucoup plus présent pour les chercheurs en sciences humaines que pour les chercheurs vétérinaires et zootechniciens, qui se bornent à reprendre les critères proposés par les premiers. Dans bien des cas, cependant, aucune distinction n'est faite, et tous les mouvements pastoraux saisonniers, pour peu qu'ils présentent une certaine régularité, sont indistinctement qualifiés de "transhumance". Mais les critères retenus dépendent du point de vue adopté.

Les géographes, dont l'intérêt s'est focalisé, parfois à l'excès, sur la caractérisation de la mobilité pastorale par son inscription spatiale, ont produit des classifications souvent un peu formelles, qui privilégient des critères liés à l'habitat et aux caractéristiques des mouvements effectués $(42,50$; pour une synthèse plus récente : 22$)$. Sont généralement considérés comme "nomades" les groupes qui ne possèdent pas d'établissement fixe et dont tous les membres se déplacent avec les troupeaux, comme "transhumants" ceux dont seulement une partie des membres se déplace, les autres demeurant en un lieu d'habitat fixe qui constitue l'un des pôles des mouvements périodiques des troupeaux, comme "sédentaires" enfin ceux qui ne se déplacent pas hors les périodes éventuelles de migration. La prise en compte du rayon d'action des déplacements, de leur périodicité, de leur régularité, de leur nombre de pôles, permet de distinguer diverses formes intermédiaires et de raffiner la classification en semi-nomadisme (entre plusieurs résidences), semi-sédentarité (durant une partie de l'année), nomadisme vertical, entre des étages altitudinaux différents, nomadisme apériodique, etc.

L'adoption d'un point de vue économique conduit à privilégier des critères liés aux activités productives et à la formation des revenus. Est ainsi qualifié de "pastoral" un système dans lequel au moins 50 p. 100 du revenu brut des unités domestiques (production commercialisée et valeur estimée de la part de la production auto-consommée) provient de l'élevage ou d'activités liées à l'élevage, et d"'agro-pastoral" un système dans lequel au moins 50 p. 100 du revenu brut provient d'activités liées à l'agriculture (production végétale) et au moins 10 p. 100 de l'élevage (60). Un critère alternatif peut être tiré de la part des calories alimentaires provenant directement de l'élevage ou d'activités connexes (61).
Les ethnologues accordent beaucoup d'importance aux critères liés à l'organisation sociale (par exemple les types de segmentation des groupes lignagers, les modèles résidentiels, la division sociale et familiale du travail, le degré de stratification sociale). A leurs yeux, l'identification d'un type de déplacernent ne permet pas de conclure à la présence d'un type particulier d'organisation sociale. Cependant, la coupure principale est entre le pastoralisme nomade et le reste, le nomadisme entraînant, du fait notamment des modèles résidentiels spécifiques dont il s'accompagne, des formes d'organisation sociale particulières et convergentes (22).

A une échelle un peu différente, BONTE et al. (16) ont proposé (voir encadré) une classification privilégiant les "faciès culturels" découlant des caractéristiques du milieu et de l'espèce animale élevée (voir aussi la classification de A. LEROI-GOURHAN, présentée par DIGARD (25 p. 222-223)).

\section{ÉLÉMENTS DE CLASSIFICATION DES SOCIÉTÉS PASTORALES}

Les sociétés pastorales sont nombreuses, occupant (seliles ou à cóté d'autres sociétés) une part énorme de la planete, et constituent ou se rattachent a des ensembles humains en apparence forts différents les uns des autres. Tres sommairement, cinq grands facles" peuvent êtro distingués

1. Les clvilisations du renne, réparties sur une partie de la Siberie et lextreme nord de l'Europe (Choukchi, Koryak, Yakout, Toungounz, Samoyèdes, Lapons, etc.).

2. Les civilisations des steppes, reposant sur l'élevage du cheval et du chameav de Bactriane. Leur diffusion s'est étendue a toute la zone eurasienne médiane, de la Hongrie a la Chine.

3. Les civilisations bedouines, qui occupent les zones arides et semi-arides du Moyen Orient et de l'Afrique saharienne (bédouins a rabes d'orient et d'Atrique Maures, Touaregs, Toubous, Somalls) Caractérisée par la domestication du dromadaire, leur relative homogenelté culturelle a été rénforcée par le polds unificateur de Hislam.

4 Les societés pastorales montagnardes localisees surtout autour du bassin méditerranéen lato sensu. en Afrique du Nord (Berberes), au Moyon-Orient (Kurdes. Pathans et autres Iraniens, Turcs) et en Europe du SUA (Saracatsans) Elles sont dominées lcl par lélevage ovin. Alleurs, le yak au tibet le lama dans les Andes ont aussi favorisé des adaptations originales.

5. Les soctétes pastorales atricaines, qui occupent une bande continue de l Atlantique a locean Indien (Peuls, Niotiques, Gallas, etc.) se prolongeant dans la zone interlacustre et en Afrique du Sud (Botswana, Hot tentots). Elles sont organisées autour de l'élevage du boeuf, parfois associé aux moutons et aux chèves, mais sans animaux de transport

(Extrait de BONTE et al (16) apres autorisation) 
Concrètement, il est possible de rechercher une combinaison de critères adaptée à chaque problématique. BONFIGLIOLI, anthropologue et africaniste, écrit ainsi, dans une étude réalisée au Tchad pour le compte de la Banque mondiale (14) : "Nous ne retenons pas du tout une classification fondée sur la distinction entre éleveurs "nomades", "transhumants", etc. En effet, d'un point de vue socio-économique, ni le mouvement, ni les modèles résidentiels ni l'habitat ne constituent, malgré leur importance, des éléments déterminants. Par contre, beaucoup plus importants sont tous les facteurs relatifs à la dépendance économique du bétail et des produits de la terre, à l'ancienneté voire à la durée historique de la pratique agricole, au type d'agriculture associée à l'élevage (culture pluviale, irriguée ou de décrue), aux spécificités du savoir technologique et aux caractéristiques générales de l'environnement physique. Bref, plus que sur des repères géographiques et résidentiels, nous nous appuyons sur des critères économiques et historiques pour identifier les formes concrètes de l'agro-pastoralisme".

En toute hypothèse, il convient d'insister sur la fluidité des limites entre les formes de pastoralisme ainsi distinguées : certains systèmes transhumants ont beaucoup de points communs avec des systèmes nomades, ces formes peuvent être associées, des groupes passent de l'une à l'autre...

\section{A PROPOS DE QUELQUES IDÉES REÇUES}

\section{Le pastoralisme nomade n'est pas la survivance d'une activité archaïque}

Le pastoralisme nomade n'est pas un stade primitif de l'évolution de nos sociétés qui, selon un schéma vulgarisé par les évolutionnistes du XIXe siècle, succéderait au stade des chasseurs-cueilleurs et précèderait une inéluctable sédentarisation agricole. Il y eut effectivement, dans certains cas, continuité entre les stades initiaux de la domestication et la spécialisation pastorale, ce qui autorise par exemple à parler, à propos du bassin méditerranéen et de l'Afrique saharienne, d'un "néolithique pastoral". Cependant, la situation la plus générale correspond au développement progressif de l'élevage dans un contexte agricole puis agro-pastoral (16). La spécialisation pastorale est consécutive, dans ce processus, à l'évolution génétique des animaux, qui s'adaptent à l'élevage en troupeaux (28), et à la croissance démographique des populations domestiques. Cette croissance est favorisée par les éleveurs dans la mesure où, au-delà de la valeur d'usage de leurs productions, les animaux ont une valeur d'échange très importante. lls représentent en effet, bien souvent, la seule forme d'accumulation disponible et se trouvent de ce fait profondément impliqués dans les stratégies sécuritaires et dans les échanges sociaux. Le développement de la mobilité pastorale, qui permet de valoriser des espaces écologiquement contrastés et complémentaires (étages altitudinaux par exemple) accompagne cette spécialisation, dont le pastoralisme nomade représente la forme ultime. Paradoxalement, celui-ci apparaît en général comme réponse et forme d'adaptation d'un système agro-pastoral sédentaire confronté à une densification du peuplement et à une crise liée à la saturation des terres agricoles.

Contre le mythe d'un Orient lointain où le monde était irrémédiablement partagé entre nomades et sédentaires (7), c'est donc d'une très ancienne dialectique entre mobilisation et sédentarisation qu'il convient de parler.

Aux yeux du zootechnicien, en dehors des rares zones où des ressources fourragères sont disponibles sur place en toute saison, il n'existe qu'une alternative à la mobilité pastorale : la constitution de réserves fourragères. Les techniques correspondantes ne débouchent sur des gains significatifs en termes de productivité du travail que dans certaines conditions, liées en particulier au milieu. Ces conditions ne sont pas toujours remplies, et la mobilité pastorale reste aujourd'hui encore l'unique formule pour exploiter les parcours des zones arides ou froides, où la période de végétation est réduite à quelques mois dans l'année. Cette mobilité et ses 'modalités concrètes, ne peuvent donc être interprétées, sur le plan technique, que par référence à la disponibilité et à l'accessibilité saisonnières des ressources pastorales, pâturages et points d'eau compris.

\section{Des pâturages moins "naturels" qu'il n'y paraît}

L'exploitation pastorale (prélèvements sélectifs, piétinement, restitutions animales, dissémination de diaspores, mise à feu) modifie profondément les écosystèmes pâturés, une partie des modifications induites étant très consciemment recherchées par les pasteurs qui "façonnent" ainsi les milieux qu'ils exploitent (17). L'évolution anthropique de ces écosystèmes pâturés n'est cependant que partiellement maîtrisée, et conduit à des formes évolutives très diverses, correspondant parfois à d'évidentes dégradations écologiques (1). Pour les sciences de l'Homme, les sociétés pastorales produisent littéralement les ressources qu'elles consomment (16). L'ensemble des connaissances et des opérations techniques que les pasteurs mettent en jeu rend insoutenable l'opposition classique entre d'une part l'élevage pastoral et la cueillette, formes d'utilisation du milieu "naturelles", passives voire prédatrices, et d'autre part l'agriculture, usage culturel et productif de ce même milieu (2)

\section{Des économies ouvertes}

Contrairement à un mythe peut-être plus tenace encore, les sociétés pastorales ne sont en aucun cas autarciques (24) ; tout au plus, les circonstances peuvent parfois les conduire à se replier momentanément sur elles-mêmes (7). Leur spécialisation productive les rend au contraire 
très dépendantes des échanges qu'elles développent avec d'autres groupes humains et principalement avec les agriculteurs sédentaires. (échange de produits de l'élevage - essentiellement des animaux sur pied - contre des condiments et des aliments glucidiques : céréales, dattes, tubercules...). La mobilité des pasteurs répond en partie à ce besoin d'échanges. De nombreux groupes pastoraux, disposant d'animaux de transport, ont même développé des activités commerciales spécialisées.

Dans le même ordre d'idées, la "rétention" supposée du bétail par les pasteurs (33: p. 51-53) est contredite par les faits d'observation (analyse de la productivité du cheptel et des régimes démographiques des populations animales domestiques) comme par l'examen attentif des données historiques : les pasteurs ont commercialisé leurs animaux depuis toujours:(31).

\section{Des pasteurs prétendument indolents}

L'élevage pastoral n'est pas une activité "contemplative", caractérisée par un faible investissement en travail. Ce jugement erroné a une double 'origine :

- une méconnaissance des techniques pastorales proprement dites : la conduite du troupeau au pâturage, par exemple, est d'autant plus efficace que les interventions du pasteur pour orienter le comportement des animaux sont plus rares et plus discrètes. Tout réside dans la relation établie entre l'homme et le troupeau, dans la vigilance et dans la finesse des observations: "le bon berger, on a l'impression qu'il ne fait rien" $(35,34: p$. 30). II faut également évoquer le travail de protection et de sauvegarde qui fait partie des tâches permanentes : protection contre les maladies, contre les fauves, contre les voleurs de bétail, contre les rivaux, lorsque les négociations n'ont pas abouti... Ce n'est pas par hasard que les pasteurs, souvent isolés, sont aussi généralement armés. Le fonçage et l'entretien des puits, l'exhaure, tâches particulièrement dures, sans cesse répétées, ne doivent pas non plus être oubliées :

- une sous-estimation du "travail social" requis par l'activité pastorale $(23: p .46)$. Ce travail recouvre deux catégories principales de tâches :

le recueil et la vérification de l'information concernant l'état des pâturages et des points d'eau, les précipitations, la situation sanitaire, la situation politique, les contraintes administratives, la sécurité des hommes, les vols de bétail, etc. En relation avec l'importance de l'information dans le travail pastoral, de nombreux auteurs ont relevé la richesse et la précision remarquables du vocabulaire technique des peuples pasteurs : les différentes catégories d'animaux, leur morphologie, leur robe, leurs comportements, leurs productions, leurs maladies, leurs apparentements, la flore (avec ses qualités alimentaires ou médicinales), les pâturages, les points d'eau, toutes les particularités du milieu physique, le climat, les pratiques d'élevage, etc. ; la conduite des négociations qui permettront de conclure et de sceller les alliances nécessaires pour accéder à l'information évoquée ci-dessus, pour ouvrir les routes et accéder aux parcours, aux résidus de récolte (par exemple dans le cadre des "contrats de fumure" conclus en Afrique entre pasteurs transhumants et agriculteurs sédentaires), à l'eau, aux marchés...

Ces "palabres inutiles", selon l'expression consacrée par l'usage colonial, représentent en fait, et contre toute apparence, des activités éminemment productives, proprement vitales pour des groupes en déplacement fréquent, économiquement très dépendants de leurs échanges.

Diverses études ont montré que la productivité du travail pastoral est élevée (19), mais il est à noter qu'elles ne prennent en compte que les opérations techniques proprement dites. Leurs conclusions méritent donc d'être nuancées, dans la mesure où elles négligent ce travail social.

\section{CARACTÈRES COMMUNS ET SPÉCIFICITÉS DES SOCIÉTÉS PASTORALES}

\section{L'élevage : un "fait social total”}

Dans les sociétés pastorales, l'animal domestique et l'activité d'élevage, très fortement et quasi exclusivement valorisée, investissent la totalité du champ économique, social et culturel. Cette valorisation exclusive a constamment frappé les observateurs et donné lieu à de très nombreux commentaires, à commencer par les célèbres développements de HERSKOVITS (29) sur le "cattle complex" des pasteurs d'Afrique de l'Est et ceux de RICHARD-MOLARD (54) sur la "boomanie" des Peuls de Guinée.

La propriété et la gestion d'un troupeau, avec tout le système de droits et d'obligations réciproques qui en découle, traduisent les liens de parenté et de voisinage. A l'inverse, toutes les solidarités, les alliances, les relations d'amitié et à plus forte raison les liens de parenté s'expriment nécessairement à travers des échanges d'animaux ou des relations de copropriété (14). La circulation sociale du bétail, seule valeur stable dont le transfert soit susceptible de cimenter les relations sociales, accompagne et matérialise l'évolution de ces relations. Le cheptel est directement impliqué dans tout processus de production ou de transmission de richesse. Le parallélisme entre constitution et développement de la famille d'une part, du troupeau 'd'autre part, a été souligné à maintes reprises $(12,16,43,44)$. 
La mobilité des pasteurs fait que l'intérêt de posséder des biens est contrebalancé par l'obligation de les transporter. En dehors des animaux, "toute richesse leur est fardeau" : propriété et mobilité sont en contradiction. Cette remarque de SAHLINS (57) s'applique tout particulièrement à la propriété foncière et l'on y reviendra.

Les sociétés pastorales sont pour la plupart des sociétés segmentaires, où la répartition des individus entre les différentes unités domestiques est régie par un principe de filiation unilinéaire, un type de mariage préférentiel et une règle de résidence. Ainsi, dans la plupart des cas, la filiation patrilinéaire s'accompagne d'une résidence patrilocale et d'une union avec la cousine parallèle patrilatérale. Ceci conduit à une fission des lignages en segments et de proche en proche à une structure d'éléments emboîtés, de l'unité la plus large (la tribu) aux plus petites (les familles nucléaires), en passant par les lignages et les clans (14). Parfois atomisées, ces sociétés sont dotées à l'échelle supérieure d'institutions politiques plus ou moins centralisées et hiérarchisées mais ne disposant souvent que de pouvoirs limités. A l'extrême, il s'agit de sociétés "émiettées et acéphales" (4). Leur fonctionnement, fondé sur l'opposition équilibrée d'unités structurellement équivalentes, s'opposerait, selon de nombreux travaux anthropologiques, à l'émergence de chefferies aux pouvoirs étendus (24:p. 99). Cette structure sociale labile est faite pour disperser, ce qui est aussi bien le moyen d'optimiser l'exploitation du milieu que de minimiser les risques, y compris politiques. Ainsi se constituent chez les Peuls d'Afrique de l'Ouest les groupes migratoires qui se font et se défont au gré de l'occupation de l'espace pastoral (26)

Enfin, des normes sociales de comportement, parfois extrêmement précises et rigides, contribuent à maintenir la cohésion des groupes en dépit de la labilité de la structure socio-politique (11).

\section{La gestion de l'espace pastoral}

La gestion collective des ressources pastorales s'oppose de manière caractéristique à la gestion domestique des troupeaux. II est d'intérêt public d'assurer l'accès aux ressources pastorales. Vu l'hétérogénéité de leur répartition dans l'espace et dans le temps, ceci a pour corollaire une appropriation collective et une gestion souple et sans exclusive, favorisant à la fois les alliances et l'atomisation-dispersion des groupes (16).

Si rien ne s'oppose à l'appropriation privée du bétail, la propriété individuelle de la terre, en revanche, entre en contradiction avec la logique des systèmes pastoraux (57). La terre ne peut pas se déplacer ni se reproduire comme le bétail. De ce fait, elle n'est pas susceptible d'une mobilité et donc d'une utilité sociale comparable à celle du bétail. En outre, la disponibilité de la terre est, par hypothèse pourrait-on dire, non limitante. Lorsque tel n'est plus le cas, lorsque la terre se fait rare, ce qui est actuellement une situation de plus en plus courante, les pasteurs sont contraints, pour faire face à cette pénurie, de définir plus strictement les conditions d'accès aux ressources et de tenter de se faire reconnaître des droits territoriaux. Bref, d'entrer dans une logique de contrôle foncier. Mais ces stratégies foncières modifient rapidement les institutions sociales et affaiblissent les solidarités collectives (14:p. 52). Elles placent ces sociétés dans des situations très difficiles, où les anciens rapports de domination s'inversent, au profit des groupes sédentaires.

\section{Le caractère guerrier}

Ce trait quasi général est lié à la fois à :

- l'exaltation du lignage qui accompagne la segmentation de l'organisation sociale, responsable de l'existence de multiples petites unités mobiles, nouant en permanence des alliances à divers niveaux, en fonction des circonstances. Une cohésion dissuasive peut néanmoins toujours être reconstituée en cas de conflit, selon le célèbre proverbe bédouin : "Moi contre mon frère ; moi et mon frère contre notre cousin ; moi, mon frère et notre cousin contre l'étranger". On a dit que le principe même du fonctionnement des sociétés segmentaires repose sur un jeu constant d'oppositions multiples d'unités de même rang. Mais cela peut aller plus loin : on peut voir dans certaines sociétés nomades de véritables clans, où la vendetta représentc un mode permanent de relations sociales, de telle sorte que la crainte des agressions l'emporte sur les autres motivations dans les rapports entre les groupes. La vendetta constitue dans ce cas un état constant, qui ne peut, contrairement à la guerre, connaître de fin $(10,27)$;

- la tendance quasi structurelle des sociétés pastorales à l'expansion (16). Elle résulte de la conjonction de caractéristiques biologiques, telles que leur propre vigueur démographique - aujourd'hui remise en cause dans nombre de cas, suite à l'évolution des conditions de vie -, le potentiel de croissance démographique de leur cheptel et le poids des contraintes écologiques $(19,52)$, et de propriétés socio-structurelles, telles que la prolifération des lignages dans les systèmes segmentaires $(47,56)$;

- la dépendance constante vis-à-vis d'autres groupes, susceptibles d'interdire l'accès à des lieux vitaux, de suspendre les échanges, de voler du bétail; d'interdire un point d'eau ...;

- la mobilité, qui joue un rôle décisif dans l'attaque comme dans la défense. La rapidité de mouvement liée à l'usage de montures est rendue plus efficace encore par la connaissance du milieu et la capacité à se disperser propre aux pasteurs, qui n'ont pas leur pareil pour s'évanouir dans la nature.

Beaucoup de peuples pasteurs ont historiquement disposé (ou disposent encore), du fait de cette valence guer- 
rière, des instruments de la domination, ce qui leur a permis de conquérir et d'exercer le pouvoir, et de diversifier à cette occasion leur insertion sociale : contrôle du commerce, de l'administration, intelligentsia. A cette occasion, ils ont parfois transmis aux populations qu'ils dominaient leur langue, leur modèle culturel, leur religion. La fulbéisation (ou fulanisation, selon un anglicisme répandu) du Nord-Cameroun en est un excellent exemple. En relation avec leur mobilité et leur caractère guerrier, les peuples pasteurs ont souvent joué un rôle éminent dans la diffusion des religions, ou des confréries musulmanes, du moins en Afrique et au Moyen-Orient.

\section{Pratiques pastorales}

\section{Vis-à-vis des animaux, une mentalité et un comportement de symbiote plutôt que de prédateur}

D'une manière générale, les pasteurs exploitent les productions animales renouvelables, lait, laine, poil et vendent des animaux vivants. Leur régime alimentaire est à base de produits laitiers et non de viande. L'abattage n'est pas banalisé : on ne sacrifie pas des animaux sains en dehors d'occasions sociales ou religieuses bien définies.

L'exploitation de la foree de travail des animaux, autre type de production renouvelable, est une constante dans les sociétés pastorales.. L'élevage des animaux de transport (bât et selle) a d'ailleurs joué un grand rôle dans la constitution de certaines d'entre elles, les sociétés pastorales turco-mongoles et bédouines notamment, en leur conférant une position privilégiée dans les échanges commerciaux et culturèls, et en contribuant à leur relative supériorité militaire (16).

\section{Le sel}

Le sel, son extraction, son commerce, sa mise à disposition des animaux (cure salée), son rôle dans la conduite des troupeaux, les vertus qui lui sont prêtées, tiennent partout une grande place dans les activités, les déplacements, les échanges et l'imaginaire des pasteurs $(6,8)$. A ce titre, le sel mérite d'être considéré comme une ressource pastorale à part entière, même s'il n'occupe pas une place aussi importante dans l'univers des zootechniciens.

\section{Le vol de bétail}

La valorisation socio-culturelle du vol de bétail est une autre constante de la culture pastorale. Certains auteurs lui attribuent même une véritable fonction sociale : redistribution, dispersion du cheptel et répartition du risque (59). Le vol est assimilé, côté pile, à un acte de bravoure, souvent chargé d'une signification initiatique (3) ; côté face, il représente un risque constant, une source non négligeable de pertes économiques, et peut devenir un véritable fléau dans certaines circonstances (20), en particulier lorsqu'il donne lieu à un brigandage organisé à grande échelle.

\section{Le feu pastoral}

L'usage du feu est attesté chez diverses sociétés de chasseurs (46), notamment chez les plus spécialisés d'entre eux, tels que les Indiens d'Amérique du Nord, chasseurs de bisons. II est très développé chez de nombreuses sociétés pastorales, notamment chez tous les pasteurs africains, les Mongols, etc. Par ses effets sur le milieu $(17,18,30,64)$, le feu joue un rôle de premier plan dans l'anthropisation des formations naturelles et l'affirmation de leur vocation.

\section{Le chien de protection}

Le chien est un acteur courant de la vie pastorale. II ne s'agit point ici, cependant, du chien de berger, mais du "chien de protection". Le chien de berger, auxiliaire de la conduite des troupeaux, généralement de plus petit format, est inconnu dans les sociétés pastorales traditionnelles. Innovation européenne récente, il n'a vu le jour, selon de PLANHOL, qu'au XVIle ou XVIIle siècle, en Scandinavie ou en Islande, d'où il a gagné les îles britanniques puis le continent européen (49). De grande taille, le chien de protection remplit pour sa part un rôle d'alerte et de défense des campements et des animaux contre les voleurs et les prédateurs. Le Chien de montagne des Pyrénées, qui protégeait des ours, ou le Mâtin des Abruzzes, qui tient les loups à l'écart, en sont deux exemples européens. Si cruciale soit sa fonction, son statut social et familial reste toujours très inférieur à celui du bétail ; souvent méprisé, mal traité, il se nourrit des déchets de la famille, et chasse occasionnellement pour son propre compte.

\section{Les techniques non pastorales. L'artisanat}

Chez les pasteurs nomades, et dans une moindre mesure chez tous les groupes plus ou moins mobiles, la culture matérielle est dominée par un impératif : tout le mobilier doit être pliable et portable, donc incassable et léger (21).

L'artisanat textile est fréquemment le seul qui soit développé chez les pasteurs nomades : la laine et le poil de diverses espèces animales (ovins, caprins, camélidés, yack...) sont utilisés pour la confection de vêtements, de tapis, de tentes. La fabrication des harnais, des tresses (en cuir ou en peau), des lanières (tissées en laine ou en poil), des cordes (parfois à base de fibres végétales), qui remplissent de multiples usages, est également assurée par les pasteurs eux-mêmes, dans la plupart des cas, à partir de leur production ou de leur cueillette. 
D'autres produits sont nécessaires à leur activité quotidienne, tels que le bois et le cuir pour les récipients destinés à contenir les produits laitiers, les outres, les barattes, les sacs indispensables lors des déplacements, le fer pour les couteaux et les armes, etc. Les pasteurs font appel pour leur fabrication au travail d'artisans, éventuellement castés, forgerons, tanneurs et autres boisseliers, qui sont économiquement, socialement et culturellement associés aux sociétés pastorales. Une tradition peule relevée au Ferlo (Sénégal) rapporte ainsi la légende de l'origine commune des Fulbé, des Lawbé (artisans du bois) et des Bambaabé (griots généalogistes), dont les ancêtres fondateurs auraient été des frères utérins (62). Dans le cas du pastoralisme nomade, ces groupes associés sont qualifiés de "nomades de service" (24).

\section{LA CRISE ET SES COMPOSANTES}

\section{La marginalisation des pasteurs}

Depuis la colonisation, les sociétés pastorales ont été intégrées à des Etats pluri-ethniques où leur rôle est rarement dominant, à l'exception de la Mauritanie, de la Somalie et de la Mongolie. Elles y sont pour la plupart confrontées à des difficultés politiques, souvent résurgences de conflits hérités d'un passé guerrier (24). Notons aussi l'hostilité des pouvoirs politiques et des administrations, qui éprouvent des difficultés à contrôler ces populations belliqueuses et insaisissables, promptes à échapper aux contraintes administratives, qu'elles soient fiscales, douanières, sanitaires... Cette situation crée un processus de marginalisation qui, à défaut d'autre mode de domination est le principe le plus caractéristique et la pratique la plus courante de l'assimilation économique et politique des pasteurs nomades dans la société globale (Ahrweiler, 1975 ; Cassin, 1975 ; Digard, 1975, cités par DIGARD (24)). Ce phénomène revêt diverses dimensions.

\section{Marginalisation économique}

Dans la plupart des cas, les groupes pastoraux ne sont pas parvenus à accéder collectivement au statut de producteurs de masse dans une économie de marché. La progressive marginalisation qui en découle est accélérée par la concurrence locale croissante des élevages sédentaires en voie d'intensification et par les très fortes distorsions de concurrence qui résultent de l'ouverture des marchés internationaux, qu'il s'agisse de viande, de peaux ou de produits laitiers. Par ailleurs, la production et l'exploitation d'animaux de transport ne conservent plus qu'un intérët local, et sont partout en déclin. De multiples indices laissent penser que l'évolution des termes de l'échange entre céréales et produits de l'élevage pastoral au cours des cinquante dernières années a été globalement défavorable, voire très défavorable pour les éleveurs, dans la plupart des situations étudiées.

\section{Marginalisation socio-culturelle}

Les populations pastorales sont défavorisées sous l'angle de l'accès aux services publics : enseignement, santé, etc., dont l'organisation est rarement pensée pour elles. Au-delà des aspects politiques, force est de reconnaître que l'organisation des services publics soulève de difficiles problèmes en milieu pastoral, en raison de la mobilité des populations et des faibles densités de peuplement.

\section{Marginalisation foncière}

Les administrations coloniales, largement influencées par les thèses évolutionnistes en vigueur à l'époque, ont quasi unanimement jugé le pastoralisme comme un mode de vie révolu, et considéré les terres des zones pastorales comme vacantes et sans maîtres. Elles ont de ce simple fait engagé un processus de marginalisation à l'encontre des sociétés concernées. Les pouvoirs nationaux issus de cette période n'ont en général pas voulu, ou pas su reconnaître aux pasteurs des droits fonciers spécifiques : en légalisant le libre accès à la terre et aux ressources pastorales, ils ont établi les conditions d'une inexorable spoliation du domaine pastoral (41). La densification généralisée de l'occupation de l'espace rural, consécutive à la croissance démographique et à l'augmentation de la productivité du travail agricole a fait le reste, en entraînant une concurrence croissante avec l'agriculture. II en résulte de multiples empiètements sur le domaine pastoral, dont l'effet vient s'ajouter à l'impact des aménagements. Les aménagements hydro-agricoles sont particulièrement redoutables pour les pasteurs, qu'ils privent de l'accès à des ressources pastorales stratégiques : pâturages de saison sèche et points d'eau $(58$, $62)$. Amputés, les parcours traditionnels sont également morcelés, ce qui rend de plus en plus problématique la circulation des troupeaux et l'accès à certaines zones pastorales. La reconnaissance de droits fonciers aux pasteurs, à laquelle divers Etats semblent actuellement se rallier de plus ou moins bon gré, se heurte à de nombreuses difficultés, et peut s'avérer un remède pire que le mal, si la définition de ces droits entre en contradiction avec leur organisation sociale et leurs pratiques coutumières (38).

\section{La dégradation écologique des zones pastorales}

Depuis longtemps les pasteurs sont dénoncés comme destructeurs de l'environnement. Mais alors que ces critiques émanaient d'administrations ou de milieux socioculturels majoritairement hostiles, depuis quelques décennies ce sont des rangs des meilleurs connaisseurs des sociétés pastorales et des pasteurs eux-mêmes que montent les cris d'alarme. 
La conjonction de la croissance des troupeaux et de l'effritement progressif de l'espace pastoral fait croître rapidement la pression exercée sur l'environnement. Le surpâturage menace lorsqu'il n'a pas encore commencé ou achevé - ses méfaits (17). Les épisodes de sécheresse qui ont marqué les dernières décennies ont aggravé et parfois tragiquement révélé la gravité du problème écologique auquel doivent faire face de nombreuses sociétés pastorales.

Les processus en cours mettent en évidence l'inadaptation croissante des stratégies sécuritaires traditionnelles, fondées sur la capitalisation du cheptel, la mobilité et l'existence aujourd'hui révolue d'"espaces de sécurité" (5). Les sociétés pastorales ne disposent plus, dans bien des cas, d'un contröle suffisant sur le milieu pour pouvoir adapter leur mode d'occupation et d'exploitation à son évolution écologique.

\section{La sédentarisation, solution définitive du problème pastoral ?}

Confrontés à des crises économiques, politiques ou écologiques, les sociétés pastorales y ont souvent répondu en renonçant temporairement à leur spécialisation productive, à leur mode de vie, à leur mobilité. L'histoire des peuples pasteurs est celle d'une dialectique permanente entre des logiques d'expansion, de pastoralisation et de spécialisation d'une part, de récession, de sédentarisation et de diversification d'autre part.

Ainsi certaines formes de sédentarisation, qui concernent les nomades appauvris, ayant perdu leur cheptel, ne constituent pas le stade ultime d'une évolution régressive irréversible du nomadisme, mais une forme transitoire d'adaptation et une condition de sa survie $(24,53)$. Des périodes de crise économique et politique peuvent donner lieu, en retour, à la conversion massive de populations villageoises au nomadisme : les "bédouinisations" médiévales du monde turco-iranien en fournissent l'exemple le plus frappant (48). Bien entendu, ces considérations historiques ne permettent nullement d'inférer l'issue de la crise généralisée actuelle du pastoralisme nomade...

Dans l'esprit de bien des responsables politiques, la sédentarisation est cependant une voie sans retour, une "solution" définitive. Comme l'écrit REBOUL (51), le postulat de la supériorité sociale de la sédentarité sur le nomadisme est partout affirmé et reconnu non comme hypothèse sociale, mais comme évidence. Considérée comme la panacée, véritable leitmotiv des politiques pastorales depuis des lustres, la sédentarisation reste le maître mot que les pouvoirs sédentaires opposent au fait pastoral. Or, qu'il trouve son origine chez les nomades ou chez les sédentaires, l'Etat est toujours appelé à devenir, tôt ou tard, sédentaire et citadin (24). Le recul historique permet pourtant aujourd'hui de conclure que ce prétendu remède entraîne généralement plus de maux qu’il n'en guérit...

\section{CONCLUSION : RISQUES ET AVENIR DES SOCIÉTÉS PASTORALES}

En relation avec leurs spécificités, les systèmes pastoraux sont donc exposés à des risques de nature particulière dont l'évolution et la hiérarchie sur l'échelle de la gravité ont été marquées au cours de l'histoire récente par les changements suivants :

- l'allègement progressif, dans les cinquante dernières années, d'une contrainte ancienne et longtemps tenue pour inéluctable: le risque sanitaire lié aux grandes épizooties, et avant tout à la peste bovine. L'apparition de la prophylaxie, en rompant cette' fatalité, a permis l'expression du potentiel de croissance des troupeaux, et a été sans aucun doute, à l'échelle mondiale, le premier facteur d'une expansion démographique sans précédent du cheptel pastoral ;

- la résurgence dans le monde post-colonial du risque politique en tant qu'événement majeur, pouvant inclure l'état de guerre ;

- le renforcement continu du risque d'une asphyxie foncière croissante, en liaison avec la marginalisation politique, économique et sociale des pasteurs ;

- la montée du risque écologique qui en résulte : de conjucturel, l'aléa climatique, dont les conséquences étaient jadis techniquement limitées et socialement gérables, se fait risque structurel en raison de la pression sur le milieu et de la disparition des espaces de sécurité. L'accélération de la dégradation des parcours et la diminution concomitante de la productivité des troupeaux en sont les conséquences, le déroulement de ce processus étant entrecoupé de crises aiguës qui viennent "réajuster" la charge des parcours en décimant les troupeaux et parfois les hommes.

La boucle est ainsi refermée. Alors sont réunis les éléments du sombre scénario qui fait tant s'interroger sur l'avenir des sociétés pastorales.

\section{BIBLIOGRAPHIE}

1. AUDRU (J.), BOUDET (G.), CESAR (J.), DULIEU (D.), GASTON (A.), MANDRET (G.), MERLIN (P.), RIPPSTEIN (G.), ROBERGE (G.), TOUTAIN (B.). Terroirs pastoraux et agro-pastoraux en zone tropicale. Gestion, aménagements et intensification fourragère. Maisons-Alfort, IEMVT, 1987. 418 p. (Etudes et Synthèses de l'IEMVT n ${ }^{24}$ )

2. BARANGER (D.) et al. L'obscure insertion d'André L. In : LANDAIS (E.) Ed. André L. Contrepoint. V'ersailles, INRA, 1991. p. 11-20 (Document de travail URSAD Versailles-Dijon-Mirecourt)

3. BAROIN (C.). Anarchie et cohésion sociale chez les Toubous. Les Daza Késerda (Niger). Paris, Editions de la Maison des Sciences de l'Homme / Cambridge University Press, 1985. 
4. BAZIN (M.). Le nomadisme Yörük aujourd'hui : les Sarikeçili du Taurus central. Prod. past. Soc., 1987, 20 : 11-29.

5. BENOIT (M.). Nature Peul du Yatenga. Remarques sur le pastoralisme en pays mossi. Paris, ORSTOM, 1982. 176 p. (Travaux et documents $n^{\circ}$ 143)

6. BERNUS (E.). Le sel du désert. Atlas Air France, 1973, 88 : 44-67.

7. BERNUS (E.). En guise de conclusion : les pasteurs nomades africains, du mythe éternel aux réalités présentes. ORSTOM, Cah. Sci. hum. , 1990 $26(1-2): 267-280$

8. BERNUS (E.), BERNUS (S.). Du sel et des dattes. Introduction à l'étude de la communauté d'In Gall et de Tegidda-n-tesemt. Niamey, CNRSH 1972. 125 p. (Etudes nigériennes $\mathbf{n}^{\circ} 31$ )

9. BERNUS (E.), POUILLON (F.) Ed. Sociétés pastorales et développement. ORSTOM, Cah. Sci. hum., 1990, 26 (1-2). 287 p. (Numéro spécial à la mémoire de Suzanne BERNUS)

10. BLACK-MICHAUD. Cohesive force. Feud in the Mediterranean and the Middle East. Oxford, Clarendon Press, 1975.

11. BOCQUENE (H.). Moi un M'Bororo. N'Doudi Oumarou, peul nomade du Cameroun. Paris, Karthala, 1986. 367 p.

12. BONFIGLIOLI (A.M.). Dudal. Histoire de famille et histoire de troupeau chez un groupe de Wodaabe du Niger. Paris, Editions de la Maison des Sciences de l'Homme/Cambridge University Press, 1988. 293 p.

13. BONFIGLIOLI (A.M.). Pastoralisme, agro-pastoralisme et retour : itinéraires sahéliens. ORSTOM, Cah. Sci. hum., 1990, 26 (1-2) : 255-266.

14. BONFIGLIOLI (A.M.). L'agro-pastoralisme au Tchad comme stratégie de survie. Essai de relation entre l'anthropologic ct la statistique. Washington D.C., Banque Mondiale/D.S.A., 1992. (Document de travail $n^{\circ} 11$ de la Banque mondiale, Enquêtes et statistiques)

15. BONTE (P.). La "formule technique" du pastoralisme nomade. In : Etudes sur les sociétés de pasteurs nomades. I. Sur l'organisation technique et économique. Paris, Cah. CNERM, 1973, 109: 6-32.

16. BONTE (P.), BRIANT (P.), BRISEBARRE (A.M.), DIGARD (J.P.) LEFEBURE (C.), LIZET (B.), ROUET (M.M.). Quatorze années de recherches sur les sociétés pastorales, texte collectif. Prod. past. Soc., $1987,20: 3-8$

17. BOUTRAIS (J.). L'élevage en Afrique : une activité dégradante ? Afr contemporaine, 1992, 161: 109-125 (numéro spécial : l'environnement en Afrique)

18. BRUZON (V.). Les savanes du Nord de la Côte-d'Ivoire, Mésologie et dynamique : l'herbe, le feu et le pâturage. Thèse de doctorat. Paris, Université Paris VII, 1990. 301 p.

19. DAHL (G.), HJORT (A.). Having herds. Pastoral herd growth and household economy. Stockholm, University of Stockholm, Department of Social Anthropology, 1976. $335 \mathrm{p}$.

20. DELAPORTE (Y.) . Les vols de bétail en Lapunie : "dun du ciel" un tragédie? In : Actes du colloque "Relations homme-animal dans les sociétés pastorales d'hier et d'aujourd'hui", Rambouillet. FAIR, 25-26 septembre 1992. Rambouillet, FAIR, 1992. p. 47-58.

21. DIGARD (J.P.). Techniques des nomades Baxtyâri d’Iran. Paris. Editions de la Maison des Sciences de l'Homme / Cambridge University Press. 1981.

22. DIGARD (J.P.). Problèmes de terminologie et de typologie des déplacements de nomades. Paris, Collège de France, 1983. p. 187-197. (Laboracements de nomades. Paris, Collège de France, 1983. p. 187-197. (Labora
toire d'ethnologie préhistorique, Séminaire sur les structures d'habitat)
23. DIGARD (J.P.) Jeux de structures. Segmentarité et pouvoir chez les nomades Baxtyâri d'Iran. L'Homme, 1987, 102: 12-53.

24. DIGARD (J.P.). Les relations nomades-sédentaires au Moyen-Orient Eléments d'une polémique. In : FRANCFORT (H.P. ) Ed. Nomades et sédentaires en Asie Centrale. Apports de l'archéologie et de l'ethnologie. Actes du colloque d'Alma-Ata (Kazakhstan), 17-26 octobre 1987. Paris, Editions du CNRS, 1990. p. 97-111.

25. DIGARD (J.P.). L'homme et les animaux domestiques : anthropologie d'une passion. Paris, Fayard, 1990

26. DUPIRE (M.). Organisation sociale des Peul, étude d'ethnographie comparée. Paris, Plon, 1970

27. EVANS-PRITCHARD (E.). The Nuer. A description of the mode of livelihood and political institutions of a Nilotic people. Oxford, Clarendon Press, 1940.

28. HELMER (D.). La domestication des animaux par les hommes préhistoriques. Paris, Masson, 1992. (coll. Préhistoire)

29. HERSKOVITS (M.J.). The Cattle Complex in East Africa. Am. Anthrop., 1926, 28 : 230-272 ; 361-380; 494-528; 633-664.

30. INNES (R.). Fire in West African vegetation. Proc, a. tall Timb. Fire Ecol. Conf., 1971, 11 : 147-173.

31. KERVEN (C.). Customary commerce : a historical reassessment of pastoral livestock marketing in Africa. London, Overseas Developmen Institute. 1992. $119 \mathrm{p}$. (Agricultural occasional paper $\mathrm{n}^{\circ} 15$ )

32. KINTZ (D.). Le foncier dans la pensée et dans la pratique des éleveurs et des agro-éleveurs. In : LE BRIS et al., Ed. L'appropriation de la terre en Afrique noire. Paris, Karthala, 1991.

33. LANDAIS (E.). Sur les doctrines des vétérinaires coloniaux français en Afrique noire. ORSTOM, Cah. Sci. hum.. 1990, 26 (1-2) : 33-71.

34. LANDAIS (E.) Ed. André L. Contrepoint. Versailles, INRA, 1991. 139 p. (Document de travail URSAD Versailles-Dijon-Mirecourt)

35. LANDAIS (E.), DEFFONTAINES (J.P.). André L. Un berger parle de ses pratiques. Versailles, INRA, 1988. 113 p. (Document de travail URSAD Versailles-Dijon-Mirecourt)

36. LANDAIS (E.), LHOSTE (Ph.), MILLEVILLE (P.). Points de vue sur la zootechnie et les systèmes d'élevage tropicaux. ORSTOM, Cah. Sc. hum. , 1987, 23 (3-4) : 421-437.

37. LE BRIS (E.), LE ROY (E.). MATHIEU (P.). L'appropriation de la terre en Afrique noire, manuel foncier APREFA. Paris, Karthala, 1991.

38. LE ROY (E.). Propriété privée ou mâttrise foncière ? Paris, Réseau Recherche-Développement / GRET, 1992. 213 p. (Dossier "élevage et gestion de terroirs")

39. LHOSTE (Ph.). Le diagnostic sur le système d'élevage. Cah. Rech. Dév. 1984 3-4 $\cdot 84-88$

40. LHOSTE (Ph.). L'association agriculture-élevage. Evolution du système agro-pastoral au Sine-Saloum (Sénégal). Maisons-Alfort, IEMVT, 1987.314 p. (Erudes et Synthèses de l'IEMVT n 21 )

41. MARTY (A.). Crise rurale en milieu nord-sahélien et recherche coopérative : l'expérience des régions de Gao et de Tombouctou (Mali, 19751982). Th. Doct. Etat. Université de Tours, 1985

42. MERNER (P.G.). Das Nomadentum in nordwestilichen Afrika. Stuttgart, Berliner Geographischen Arbeiten, 1937.

43. MILLEVILLE (P.). Une méthode d'approche du rôle social de l'élevage dans un milieu sahélien : l'enquête généalogique sur le bétail. In : 
LANDAIS (E.) Ed. Méthodes pour la recherche sur les systèmes d'élevage en Afrique intertropicale. Maisons-Alfort, IEMVT/ISRA, 1986. p. 167177. (Ftudes et synthèses de l'IEMVT n ${ }^{\circ} 20$ )

44. MILLEVILLE (P.). COMBES (J.), MARCHAL (J.). Systèmes d'élevage sahéliens de l'Oudalan. Etude de cas. Ouagadougou, ORSTOM, 1982. $127 \mathrm{p}$. + annexes

45. OXBY (C.). Peuples pasteurs en crise, les réponses des organisations non gouvernementales en Afrique. Paris, ACORD, Syros alternatives, 1990. 141 p. (coll. Ateliers du développement-GRET-FPH)

46. PERLÈS (C.). Préhistoire du feu. Paris, Masson, 1977. 180 p.

47. PETERS (E.L.). The proliferation of segments in the lineage of the Bedouin of the Cyrenaica. $J l R$. Anthrop. Inst., 1960, $90: 29-53$.

48. PLANHOL (X. de). Les fondements géographiques de l'histoire de 1'Islam. Paris, Flammarion, 1968. 442 p.

49. PLANHOL (X. de). Le chien de berger : développement et signification géographique d'une technique pastorale. Bull. Ass. Géog. fr., 1969, 370 : 355-368.

50. PLANHOL (X. de), ROGNON (P.). Les zones tropicales arides et subtropicales. Paris, Armand Colin, 1970. p. 252-289;371-379.

51. REBOUL (C.). Danger d'oasis ? Aléas d'une politique de sédentarisation. Le forage de Labgar au Sénégal. Civilisations, 1978, XXVIII (1-2) : 120-139.

52. REDDING Jr (R.W.). Decision making in subsistence herding of sheep and goats in the Middle East. Ph. D. Chicago, University of Michigan, 1981 .

53. RETAILLE (D.). Le destin du pastoralisme nomade en Afrique. Inf. géogr., 1989, 53 : 103-113.

54. RICHARD-MOLARD (J.). L'Afrique Occidentale Française. Paris, Berger-Levraut, 1949.

DIGARD (J.-P.), LANDAIS (E.), LHOSTE (Ph.). The crisis of pastoral societies. A multidisciplinary analysis. Revue Élev. Méd. vét. Pays trop., 1993, $46(4): 683-692$

This paper was prepared from exchanges between an anthropologist, who is a specialist of pastoral societies, and two specialists in anima production around the theme: the future of shepherd people. The purpose was to provide the reader with a reference document for the analysis of local effects of the generalized crisis of pastoral societies throughout the world. After having defined the "pastoral fact" and recalled the bases of the classification of the different forms of pastoralism, the authors refute some settled ideas: nomadic pastoralism is not the survival of an archaic activity, but a technically pertinent adaptation to the environment; the plants of the exploited rangeland are mostly anthropical and hence their reproduction depends on the maintenance of pastoralism; pastoral societies are not autarcic, but they use an open economy; the so-called "indolence" of shepherds is a legend due to lack of knowledge of pastoral work. The authors give a short survey of the common traits and specificities of pastoral societies (the central place of husbandry in the economic, social and cultural field, the warrier trait, the modes of range management and pastoral practises) before describing two processes which play a major part in the present crises, i.e. the political, economic, socio-cultural and territorial marginalisation of shepherds and the ecological degradation of pastoral areas.

Key words : Pastoralism - Pastoral society - Breeder - Pasture - Rangelands - Transhumance - Nomadism - Farming system - Livestock economics - Environments.
55. ROJAT (D.). Pâturages communs : modélisation bio-économique et gestion des systèmes pastoraux. Maisons-Alfort, IEMVT, 1991. $60 \mathrm{p}$. (Etudes et Synthèses de l’IEMVT n³7)

56. SAHLINS (M.). The segmentary lineage : an organization of predatory expansion. Am. Anthron., 1961, 63 (2) : 322-345.

57. SAHLINS (M.). La première société d'abondance. Temps mod., 1968, 24 (268) : 641-680.

58. SANTOIR (C.). Raison pastorale et politique de développement : les Peul sénégalais face aux aménagements. Paris, ORSTOM, 1983 (Travaux et Documents de l'ORSTOM, $\mathrm{n}^{\circ} 166$ )

59. SWEET (L.E.). Camel raiding of North Arabian Bedouin : a mechanism of ecological adaptation. Am. Anthrop., 1'965,67 : 1132-1150.

60. SWIFT (J.J.). West African Pastoral Production System. Ann Arbor, University of Michigan, Center for research on Economic Development, 1979

61. SWIFT (J.J.), WILSON (R.T.) HARMSWORTH (J.). Les systèmes de production animale au Mali. In : WILSON (R.T.), LEEUW (P.N. de), HAAN (C. de). Ed. : Recherches sur les systèmes des zones arides du Mali : résultats préliminaires. Addis-Abeba (Ethiopie), CIPEA, 1983 p. 8-18. (Rapport de recherche $\mathrm{n}^{\circ} 5$ )

62. TOURE (O.), ARPAILLANGE (J.). Peul du Ferlo. Paris, L'Harmattan, $1986.78 \mathrm{p}$.

63. VAN OPSTAL (M.), NEANTt (P.) éd. Entre tradition et modernité, quel avenir pour les sociétés pastorales ? Actes du colloque "Relations homme-animal dans les sociétés pastorales d'hier et d'aujourd'hui', Rantbouillet, FAIR, 25-26 septembre 1992. Rambouillet, FAIR, 1992. 226 p.

64. WEIN (R.W.), MAC LEAN (D.A.). The Role of Fire in Northern Circumpolar Ecosystems. London. 1983.

DIGARD (J.-P.), LANDAIS (E.), LHOSTE (Ph.). La crisis de las sociedades pastorales desde un punto de vista pluridisciplinario. Revue Élev. Méd. vét. Pays trop., 1993, 46 (4) : 683-692

Esta investigación es el fruto de un intercambio entre un antropólogo, especialista de las sociedades pastorales, $y$ de dos zootécnicos, alrededor del tema del futuro de los pueblos de pastores. La meta es ofrecer al lector un marco de referencia para el análisis de las manifestaciones locales de la crisis generalizada de las sociedades pastorales alrededor del mundo. Después de definir el "hecho pastoral" y de recordar las bases de la clasificación de las diferentes formas de pastoralismo, los autores rechazan algunas ideas concebidas : el pastoralismo nómada no es el sobreviviente de una actividad areaica, sino una forma de adaptación, que conserva su pertinencia técnica; las formaciones vegetales de las tierras de pastos explotadas son generalmente antrópicas y el mantenimiento del pastoralismo es la condición para su reproducción; las sociedades pastorales, lejos de ser autárquicas, reposan sobre una economía abierta; la pretendida "apatía" de los pastores es un mito que nace de un desconocimiento del trabajo pastoral. Se presenta un panorama rápido de las características comunes y de las especificidades de las sociedades pastorales (el lugar central de la crianza en el campo económico, social y cultural ; el carácter guerrero; las modalidades de gestión del espacio y de las prácticas pastorales), para luego describir dos procesos que juegan un papel mayor en la crisis actual: la marginalización política, económica, socio-cultural y territorial de los pastores; la degradación ecológica de las zonas pastorales.

Palabras claves : Pastoralismo'- Sociedad pastoral - Ganadero - Pasto - Tierras de pastos - Transhumancia - Nomadismo - Sistema ganadero Economía de la cría - Medio ambiente. 